\title{
Editorials \\ Recent advances in schizophrenia research
}

Editoriali

Recenti progressi nella ricerca sulla schizofrenia

\author{
MICHELE TANSELLA, Editor
}

Research on schizophrenia, aiming to achieve a better understanding of this severely disabling disorder, is in a florid phase. Different approaches, from genetic to clinical, psychosocial and environmental, are producing interesting, even if not always converging results, that will hopefully generate new insights into the pathogenesis of this disorder and new hopes for a better treatment and care of the people affected and their families.

This issue of EPS includes three Editorials on recent advances in schizophrenia research, by leading scientists presently working in Australia, Europe and USA.

Assen Jablensky from Perth discusses the "Long and winding road of schizophrenia research", reminding us that, in spite of the availability of an increasing number of novel technologies, including molecular genetics and functional neuroimaging, no major breakthrough has yet occurred. We need to avoid, in future research, following several specific pathways by investigating selected, single and isolated features of this complex disorder, and we need not losing the "big picture" of the disease. The current diagnostic systems (ICD 10 and DSM-IV) do not provide the most appropriate phenotype for aetiological research, and studies attempting to re-focus the phenotype definition on more homogeneous group of symptoms (example, the "deficit syndrome") are welcome. After a brief summary of recent research conducted in Australia by his research team, Jablensky points out his view on the possibility that the clinical manifestations of schizophrenia may represent a "common final pathway" for many different brain disorders (schizophrenia variants), with distinct genetic underpinnings. Identifying the genetic basis of latent phenotypes that are primarily expressed as neurocognitive or neurophysiological deficit may be the task for the future.

Marta Di Forti and Robin Murray from London in their Editorial on "Cannabis consumption and risk of
La ricerca sulla schizofrenia, finalizzata ad una migliore comprensione di questo disturbo altamente disabilitante, è in una fase florida. Diversi approcci, da quello genetico a quello clinico, psicosociale ed ambientale, stanno producendo risultati interessanti, anche se non sempre convergenti, che ci auguriamo produrranno nuovi insight nella patogenesi di questo disturbo che ci consentano di poter meglio trattare ed assistere i pazienti e le loro famiglie.

Questo numero di EPS include tre Editoriali, scritti da ricercatori di primo piano che lavorano in Australia, Europa e USA.

Assen Jablensky di Perth, nel primo Editoriale intitolato "Long and winding road of schizophrenia research", ci ricorda che, nonostante sia oggi disponibili un crescente numero di nuove tecnologie, tra cui la biologia molecolare ed il neuroimaging funzionale, non ci sono ancora stati grandi progressi. Dobbiamo evitare, in futuro, di seguire percorsi di ricerca troppo specifici, indagando su aspetti selettivi, singoli e isolati di questo disturbo complesso, perdendo di vista il quadro generale della malattia. I sistemi diagnostici attuali (ICD 10 e DSM-IV) non forniscono la descrizione del fenotipo più appropriato per condurre ricerche etiologiche e studi che rimettano a fuoco una definizione di fenotipo della schizofrenia, applicabile ad un gruppo più omogeneo di sintomi (ad esempio la "sindrome deficitaria"), sarebbero preferibili. Dopo aver riassunto brevemente i risultati di ricerche recenti, condotte da lui e dal suo gruppo in Australia, Jablensky esprime il parere che le manifestazioni cliniche della schizofrenia possano rappresentare un pathway finale comune per molti, diversi disturbi del cervello (varianti della schizofrenia) con sottostanti distinte caratteristiche genetiche. L'obiettivo per il futuro potrebbe essere allora quello di identificare le basi genetiche di fenotipi latenti che si manifestano con deficit neurocognitivi e neurofisiologici. 
developing schizophrenia: myth or reality?" summarise the most recent data on the association between cannabis use and schizophrenia. A number of prominent studies have strengthened our understanding of that association and demonstrated that cannabis use, especially during adolescence, increases the subsequent risk of developing schizophrenia. However, only a small proportion of users become psychotic. The evidence shows that the individuals who are genetically predisposed to psychosis (e.g. have an affected relative) are at increased risk. After a critical appraisal of the epidemiological, pharmacological and genetic studies addressing the association between cannabis use and schizophrenia, they conclude that the available data suggest that cannabis plays a modest role in the aetiology of schizophrenia as a component cause; it is part of a constellation of causes including predisposing genes, obstetric events, and probably some still undetermined causes.

Finally Matcheri Keshavan and his collaborators from Detroit, Michigan remind us that major psychiatric disorders beginning in adolescence, namely schizophrenia, bipolar disorders and obsessive compulsive disorders (OCD) frequently coexist, and are preceded by similar premorbid characteristics. In their Editorial "Developmental biomarkers in schizophrenia and other psychiatric disorders: Common origins, different trajectories", after reviewing the literature, with a particular focus on neuroimaging studies, they present a conceptual model to integrate these disorders in a developmental framework. They conclude that frontostriatal alterations may account for the premorbid attentional and behavioural disorders that often overlap across schizophrenia, bipolar disorder and OCD. Disorders of affect and obsessive compulsive behaviours that emerge later in these disorders may stem from maladaptive neuroplastic changes in limbic brain structures.

We shall find the most productive way, in conducting further research for attempting to solve, at least partially, one of the biggest enigmas in medicine: understanding schizophrenia. It is likely that this attempt would be more fruitful if coordinated efforts try in future to explore together biological and environmental factors, and their interactions, within a coherent theoretical paradigm. We need the bio-psycho-social approach not to become a bio-bio-bio approach.
Marta Di Forti e Robin Murray, di Londra, nel loro Editoriale intitolato "Cannabis consumption and risk of developing schizophrenia: myth or reality?", riassumono i dati più recenti sull'associazione tra uso di cannabis e schizofrenia. Un buon numero di studi importanti hanno aumentato le nostre conoscenze su questa associazione, dimostrando che l'uso di cannabis, specialmente durante l'adolescenza, aumenta il rischio di sviluppare poi schizofrenia. Tuttavia noi sappiamo che solo una piccola parte degli utilizzatori di cannabis diventano poi psicotici. Le evidenze dimostrano tuttavia che gli utilizzatori geneticamente predisposti alle psicosi (ad esempio coloro che hanno un familiare schizofrenico) hanno un rischio più elevato. Dopo aver analizzato criticamente gli studi epidemiologici, farmacologici e genetici che si sono occupati dell'associazione tra uso di cannabis e schizofrenia, gli Autori concludono che le evidenze disponibili suggeriscono che la cannabis, pur avendo un ruolo causale modesto nell'eziologia della schizofrenia, fa parte di una costellazione di fattori causali che comprendono geni predisponesti, eventi ostetrici e probabilmente altre cause ancora non note.

Infine Matcheri Keshavan ed i suoi Collaboratori di Detroit, Michigan ci ricordano che disturbi psichiatrici maggiori ad esordio nell'adolescenza, come la schizofrenia, il disturbo bipolare ed il disturbo ossessivo-compulsivo frequentemente coesistono e sono preceduti da caràtteristiche premorbose simili. Nel loro Editoriale intitolato "Developmental biomarkers in schizophrenia and other psychiatric disorders: Common origins, different trajectories", dopo una revisione della letteratura, in particolare di quella sugli studi di neuroimaging, essi presentano un modello concettuale che integra questi disturbi in un quadro comune, un disturbo del neurosviluppo. Essi poi concludono che alterazioni fronto-striatali possono spiegare i disturbi premorbosi dell'attenzione e del comportamento, che spesso sono comuni a schizofrenia, disturbo bipolare ed OCD. I disturbi dell'affettività ed i comportamenti ossessivi-compulsivi che si osservano più tardi possono invece derivare da cambiamenti neuroplastici maladattativi nelle strutture del sistema libico.

Nelle ricerche future, per tentare di risolvere, almeno parzialmente, quello che è uno dei più grandi enigmi della medicina, vale a dire la comprensione della schizofrenia, sarà necessario trovare la strada giusta. È verosimile che questo tentativo sarà più fruttuoso se sforzi coordinati cercheranno di valutare al tempo stesso fattori biologici e fattori ambientali, insieme alle loro interazioni, all' interno di una paradigma teoretico coerente. Abbiamo bisogno che l'approccio bio-psico-sociale non diventi un approccio biobio-bio. 